\title{
CS06-02
}

\section{DOES THE FIRST EXPOSURE TO ADDICTIVE SUBSTANCES PREDICT FUTURE DEPENDENCE?}

\section{J. DiFranza}

Family Medicine and Community Health, University of Massachusetts Medical School, Worcester, USA

Aims: The risk factors for trying a cigarette are well known, however we were interested in the factors that determine which youths become addicted to nicotine once they have tried it.

Method: To investigate this we followed a cohort of 1246 students (mean baseline age of 12.2 years) over 4 years. Subjects underwent 11 interviews during which we assessed 45 risk factors, measured diminished autonomy over tobacco with the Hooked On Nicotine Checklist, and evaluated tobacco dependence using the International Classification of Diseases-10 ${ }^{\text {th }}$ revision. Cox proportional hazards models were used.

Results: Among 217 youths who had inhaled from a cigarette, the loss of autonomy over tobacco was predicted by feeling relaxed the first time inhaling from a cigarette (adjusted Hazard Ratio $(H R)=3.26 ; 95 \% \mathrm{Cl}, 1.95-5.46 ; \mathrm{P}<.001$ ) and depressed mood $(H R=1.29 ; 1.09-1.54 ; P=.004)$. Tobacco dependence was predicted by feeling relaxed $(H R=2.43 ; 1.27-4.65 ; P=.007)$, familiarity with Joe Camel $(H R=2.19 ; 1.11-4.32 ; P=.02)$, novelty seeking $(H R=1.56 ; 1.06-2.29 ; P=.02)$, and depressed mood $(H R=1.17 ; 1.04-1.30$; $\mathrm{P}=.007)$.

Conclusion: Once exposure to nicotine had occurred, remarkably few risk factors for smoking consistently contributed to individual differences in susceptibility to the development of dependence. An experience of relaxation in response to the first dose of nicotine was the strongest predictor of both dependence and lost autonomy. This association was not explained by trait anxiety or many other psychosocial factors. These results are discussed in relation to the theory that addiction is initiated by the first dose of nicotine. 